\title{
APLICAÇÃO DE BLOCKCHAIN NA INTERNET DAS COISAS: UMA ANÁLISE BIBLIOMÉTRICA
}

\author{
Lucas Coelho de Avila \\ Instituto Federal Fluminense \\ Rua Dr. Siqueira, 273 - Pq. Dom Bosco - Campos dos Goytacazes \\ lcoelho_avila@hotmail.com \\ Fernanda Campos de Araújo \\ Instituto Federal Fluminense \\ Rua Dr. Siqueira, 273 - Pq. Dom Bosco - Campos dos Goytacazes \\ fernanda.campos.araujo@gmail.com \\ Luiz Gustavo Lourenço Moura \\ Instituto Federal Fluminense \\ Rua Dr. Siqueira, 273 - Pq. Dom Bosco - Campos dos Goytacazes \\ prof.luizgustavo.iff@gmail.com \\ Henrique Rego Monteiro da Hora \\ Instituto Federal Fluminense \\ Rua Dr. Siqueira, 273 - Pq. Dom Bosco - Campos dos Goytacazes \\ dahora@gmail.com
}

\begin{abstract}
RESUMO
Nos tempos modernos, novas tecnologias vem surgindo com o intuito de promover mudanças e revolucionar o modo de viver da sociedade. Uma tecnologia bastante promissora é a IoT, que pode ser entendida como uma rede capaz de conectar inúmeros objetos à internet, podendo ser aplicada à diversas áreas, facilitando a realização de atividades e gerando benefícios para as pessoas e para as empresas. Outro conceito inovador bastante recente é o Blockchain, que funciona como um órgão centralizador, capaz de armazenar as transações que ocorrem em uma determinada rede; por isso, pode ser utilizado em inúmeras aplicações onde haja necessidade de garantia e confiança na comunicação entre partes. Este presente trabalho apresenta como objetivo realizar um estudo bibliométrico da produção científica indexada na base de dados Scopus, a respeito do tema aplicações de Blockchain em IoT. Para tal, foram definidas as palavras-chave juntamente com seus Tesauros, e após isso foram realizadas pesquisas com esses tesauros, de forma separada e de acordo com cada intersecção possível entre eles. As pesquisas foram efetuadas considerando todos os trabalhos encontrados até o ano de 2018, e os resultados foram analisados segundo o ano de publicação, principais autores identificados, principais áreas de publicação, principais países e afiliações. Foram elaborados gráficos e diagramas para facilitar as análises. Os resultados encontrados indicam que o tema possui uma trajetória crescente do número de publicações,
\end{abstract}


os Estados Unidos é o país responsável por maior quantidade de trabalhos publicados, a China o país que detém o maior número de afiliações e a Ciência da Computação a área responsável por maior quantidade de pesquisas. Pode-se concluir que o tema é recente e bastante promissor, apresentando tendências de crescimento e possuindo aplicabilidade em diversas áreas.

Palavras-chave: Estudo bibliométrico; Blockchain; Internet das coisas; Aplicações.

\begin{abstract}
In modern times, new technologies have been emerging in order to promote change and revolutionize the way of life of society. A very promising technology is IoT, which can be understood as a network capable of connecting numerous objects to the Internet, and can be applied to various areas, facilitating activities and generating benefits for people and businesses. Another very recent innovative concept is Blockchain, which acts as a centralizing body, capable of storing the transactions that occur on a given network; Therefore, it can be used in many applications where there is a need for guarantee and confidence in communication between parties. This paper presents the objective of conducting a bibliometric study of scientific production indexed in the Scopus database, regarding the theme of Blockchain applications in IoT. To this end, the keywords were defined along with their Thesaurus, and after that searches were conducted with these Thesaurus, separately and according to each possible intersection between them. The researches were performed considering all the works found until 2018, and the results were analyzed according to the year of publication, main authors identified, main areas of publication, main countries and affiliations. Graphs and diagrams were made to facilitate the analysis. The results indicate that the theme has a growing trajectory of publications, the United States is the country responsible for the largest amount of published works, China is the country that has the largest number of affiliations and Computer Science the area responsible for greater amount of research. It can be concluded that the theme is recent and very promising, presenting growth trends and having applicability in several areas.
\end{abstract}

Keywords: Bibliometric study; Blockchain; Internet of things; Applications.

\title{
Como Citar:
}

AVILA, Lucas Coelho de; ARAÚJO, Fernanda Campos de; MOURA, Luiz Gustavo Lourenço; DA HORA, Henrique Rego Monteiro. Aplicação de Blockchain na Internet das Coisas: uma Análise Bibliométrica. In: SIMPÓSIO DE PESQUISA OPERACIONAL E LOGÍSTICA DA MARINHA, 19., 2019, Rio de Janeiro, RJ. Anais [...]. Rio de Janeiro: Centro de Análises de Sistemas Navais, 2019.

\section{INTRODUÇÃO}

Blockchain é um banco de dados distribuído, online, público e que pode ser atualizado por qualquer nó participante da rede peer-to-peer (P2P) baseado no consenso entre eles e assegurado por um algoritmo de uma prova de trabalho (Proof-of-Work), que tem 
o objetivo principal de dissuadir ataques cibernéticos (KYPRIOTAKI; ZAMANI; GIAGLIS, 2015).

A Internet das Coisas (IoT) nasce da interconexão entre dispositivos dotados de computação embarcada e unicamente identificados através da infraestrutura existente da Internet, oriunda da oferta de conectividade avançada de dispositivos, sistemas e serviços que vão além da comunicação M2M, atendendo a uma variedade de protocolos, domínios e aplicações (Höller et al., 2014).

Internet das Coisas e Blockchain são consideradas tecnologias emergentes na atualidade. Ao mesmo tempo que transformam conceitos e criam novas possibilidades, cada uma em seus respectivos cenários, existe a oportunidade de desenvolver aplicações que capazes de compartilhar as características intrínsecas de ambos. Dessa forma a IoT pode se beneficiar da natureza descentralizada da Blockchain (CHICARINO et al., 2017).

De acordo com Chicarino et al. (2017) devido à sensibilidade de seus serviços, as soluções de IoT devem abordar as preocupações de segurança e privacidade em torno dos dispositivos e dos dados que coletam, geram e processam. Geralmente, seus principais cenários encontram-se no domínio financeiro, onde a Blockchain oferece diversas possibilidades de aplicações, podendo ser utilizada para resolver problemas de segurança e privacidade.

A bibliometria é uma técnica quantitativa e estatística para medir índices de produção e disseminação do conhecimento, bem como acompanhar o desenvolvimento de diversas áreas científicas e os padrões de autoria, publicação e uso dos resultados de investigação (LOPES et al. 2012).

Tendo em vista a relevância dessa temática para o mundo moderno atual, no qual o ambiente digital vem ganhando cada vez mais espaço, essa pesquisa possui como objetivo mapear a produção científica na área de Blockchain aplicada a internet das coisas. Propõe-se identificar como se encontra esta temática de pesquisa no meio científico, e oferecer a potenciais pesquisadores da área, informações relevantes para estudos sobre esta matéria.

\section{REVISÃO DE LITERATURA}

A Blockchain surgiu através da criptomoeda Bitcoin, tendo por objetivo atuar como se fosse um livro-razão capaz de armazenar as transações financeiras de todos os usuários de Bitcoin de uma forma organizada e segura, não havendo a necessidade de um órgão centralizador para validar as transações realizadas. Percebeu-se que esse conteúdo poderia ser utilizado em variados tipos de aplicações na tentativa de evitar adulterações, tais como, contratos, eleições digitais, aplicações com necessidade de garantia contra erros e modificações, armazenamento de dados, etc. (LUCENA; HENRIQUES, 2016).

Segundo Jesus et al. (2018), a Blockchain é também conhecida como protocolo da confiança, sendo um conceito que visa a descentralização como medida de segurança. Pode ser entendida como bases de registros e dados distribuídos e compartilhados que possuem a função de criar um índice global para todas as transações que ocorrem em uma determinada rede. Sua função se assemelha a de um livro-razão, porém, funciona de forma pública, compartilhada e universal, criando consenso e confiança na comunicação direta entre duas partes. 
De acordo com Xu et al. (2018) a tecnologia já vem sendo amplamente utilizada por empresas ou organizações como um meio de reorganizar redes centralizadas, haja visto sua natureza descentralizada. Uma blockchain ao funcionar como um banco de dados armazena transações como um conjunto de registros ordenados por tempo. Então, as transações são agrupadas em blocos, formando uma cadeia de criptografia em uma rede descentralizada.

A Blockchain possui um conceito inovador e o impacto previsto com a sua utilização pode ser comparado aos efeitos relacionados ao surgimento da Internet e das mídias sociais. De acordo com Lucena e Henriques (2016) a tecnologia pode ser utilizada em diversos tipos de aplicações, tais como, em armazenamento de dados, distribuição de mídias, votação eletrônica, identificadores pessoais, etc. Uma das possíveis utilizações que vem gerando grandes expectativas é com relação à Internet das Coisas (IoT), outro conceito inovador cujo crescimento é cada vez maior na atualidade.

Internet das Coisas é um termo abrangente e se refere aos esforços para conectar uma grande variedade de coisas físicas às redes de comunicação. Atualmente é crescente o número de equipamentos conectados à Internet, tais como TV's, computadores, celulares, smartphones, eletrodomésticos, etc. O Cisco Internet Business Solutions Group (IBSG) estima que até 2020 mais de 50 bilhões de dispositivos estarão conectados, o momento exato em que estariam conectados à internet mais "coisas ou objetos" do que pessoas (EVANS, 2011).

Para alcançar um crescimento tão grande, é necessário padronizar protocolos e criar as camadas adequadas para uma arquitetura que fornecerá serviços para dispositivos IoT. Atualmente, a maioria das soluções de IoT dependem do paradigma servidor-cliente centralizado, conectando-se a servidores na nuvem através da Internet. Embora esta solução possa funcionar atualmente, o crescimento esperado sugere que novos paradigmas terão que ser propostos. Entre essas propostas, arquiteturas descentralizadas foram sugeridas no passado para criar redes de sensores sem fio peer-to-peer (P2P), mas algumas partes estavam faltando em relação à privacidade e segurança até a chegada da tecnologia blockchain (FERNÁNDEZ-CARAMÉS; FRAGA-LAMAS, 2018).

A Blockchain pode contribuir para a privacidade e a segurança na internet das coisas. Neste contexto, a tecnologia pode ser utilizada para autenticar, autorizar, e auditar os dados gerados pelos dispositivos. Devido a sua natureza descentralizada, diminui a possibilidade de falhas e aumenta a eficiência do processo (JESUS et al., 2018).

O trabalho proposto por Lee e Kim (2018) introduziu uma Blockchain para evitar ameaças à segurança, como a falsificação de dados, o que poderia ocorrer usando medidores inteligentes. O Protocolo de conhecimento-nulo, uma tecnologia de melhoria de anonimato em cadeia Blockchains, foi introduzido para prevenir ameaças à segurança, como violação de informações pessoais por meio de consulta em bloco. Foi proposto o uso de contratos inteligentes para evitar falsificação de dados de medidores inteligentes e infração de informações pessoais.

JEON, KIM e KIM (2018) utilizaram uma blockchain na plataforma IoT e uma versão de cógido aberto da Ethereum (plataforma descentralizada capaz de executar contratos inteligentes e aplicações descentralizadas usando a tecnologia blockchain), para armazenar e gerenciar dados de sensores em tempo real. Os autores propõem uma nova plataforma aberta de servidor IoT que não use o servidor Mysql, devido a sua 
vulnerabilidade. Também propuseram um método e uma estrutura para armazenar dados do sensor em tempo real em um bloco, usando o contrato inteligente na plataforma Ethereum.

Um dos desafios técnicos de ter bilhões de dispositivos implantados em todo o mundo é a capacidade de gerenciá-los. Novo (2018) apresenta uma nova arquitetura para gerenciar dispositivos IoT. A arquitetura fornece um sistema de controle de acesso descentralizado conectado a redes de sensores distribuídas geograficamente. A solução é baseada na tecnologia Blockchain, enquanto as políticas de controle de acesso são aplicadas por ela. Ao adotar a Blockchain, essa solução elimina o gerenciamento de acesso centralizado.

Segundo Leal (2017) na IoT, independente do setor da economia, a Blockchain pode proporcionar uma forma de rastrear a história única de cada dispositivo, registrando a troca de dados entre ele e outros dispositivos, serviços web e usuários humanos. Pode também permitir que dispositivos inteligentes se tornem agentes independentes que conduzem de forma autônoma uma variedade de transações.

\section{METODOLOGIA}

A metodologia para elaboração desse trabalho consiste na realização de um estudo bibliométrico com o objetivo de identificar os trabalhos na área de Blockchain aplicada a internet das coisas, bem como de apresentar uma visão da produção científica nacional e internacional dentro deste contexto. É possível destacar os principais autores, artigos e periódicos relacionados ao tema e também analisar a quantidade de publicações relacionadas, de acordo com cada ano em particular, e assim perceber como tem sido os avanços nos estudos e pesquisas relacionadas ao assunto. Os procedimentos que foram executados nesse estudo corresponderam aos passos propostos por Costa (2010):

[1] Definição da amostra;

[2] Definição das palavras-chave e tesauros;

[3] Pesquisa na base de dados selecionada;

[4] Coleta de dados;

[5] Organização dos resultados em diagrama específico;

[6] Análise dos resultados.

A base de dados definida como amostra para a realização do estudo bibliométrico foi a Scopus ${ }^{\circledR}$. Justifica-se a escolha da base Scopus ${ }^{\circledR}$ por ser considerada hoje a maior base de dados multidisciplinar de resumos, citações e textos completos da literatura científica mundial, lançada pela Editora Elsevier, em 2004 (OLIVEIRA; GRÁCIO, 2013). A Scopus ${ }^{\circledR}$ oferece ferramentas inteligentes para rastrear, analisar e visualizar a pesquisa, fornecendo uma visão abrangente da produção mundial de pesquisa nas áreas de ciência, tecnologia, medicina, ciências sociais e artes e humanidades (REDONDO et al., 2017).

Para a realização da pesquisa foram definidas como palavras-chave: blockchain, internet das coisas, aplicações, e também os tesauros e termos que as representam na literatura. Em seguida, foi realizado o levantamento na base Scopus ${ }^{\circledR}$, tendo sido 
considerados todos os documentos encontrados até o ano de 2018. Por último, foi realizada a coleta de dados com o intuito de realizar uma análise da evolução cronológica da produção científica, dos trabalhos e autores mais citados e das fontes de publicação.

As palavras-chave mencionadas, juntamente com seus tesauros e termos, encontramse indicados na Tabela 1.

Tabela 1: Termos utilizados para pesquisa

\begin{tabular}{|c|c|}
\hline Tesauros de A & Blockchain \\
\hline Tesauros de B & $\begin{array}{c}\text { Iot OR Internet of Thing* OR } \\
\text { Internet of Everything }\end{array}$ \\
\hline Tesauros de C & Applic* \\
\hline \multicolumn{2}{|c|}{ Fonte: Elaborado pelos autores }
\end{tabular}

Para a realização das buscas, os Tesauros de A (Blockchain) e de B (IoT) primeiramente foram pesquisados de forma isolada na base de dados, na tentativa de verificar como vem ocorrendo os avanços nas publicações relacionadas a cada um dos assuntos. Após isso, os três termos que compõem o tema, juntamente com seus Tesauros, foram pesquisados concomitantemente com as intersecções possíveis entre eles: A e B; A e C; B e C; A, B e C. O termo C por ser mais genérico, não foi analisado de forma isolada.

Os resultados das pesquisas para os termos A e B e para cada intersecção foram agrupados das seguintes maneiras: total de resultados, número de resultados para cada ano, principais autores encontrados, principais áreas das publicações. Foram elaborados gráficos e tabelas para facilitar a análise dos resultados.

\section{RESULTADOS E DISCUSSÃO}

Segundo Ravelli et al. (2009), os estudos bibliométricos ajudam a conhecer e avaliar a produtividade e qualidade das pesquisas relacionadas a determinado assunto, permitindo determinar tendências e padrões de comportamento. Essas informações facilitam a compreensão de como o conhecimento científico vem sendo difundido e incorporado, por isso as análises bibliométricas vêm sendo cada vez mais requisitadas para várias finalidades, tais como, quantificação da produção e identificação de grupos e áreas de excelência acadêmica.

Após realizado o estudo bibliométrico na Base Scopus ${ }^{\circledR}$ os resultados obtidos encontram-se detalhados e analisados abaixo, de acordo com a metodologia proposta.

\subsection{TOTAL DE TRABALHOS ENCONTRADOS}

Através do número de trabalhos encontrados para cada pesquisa, pode-se descobrir quais os termos que geram maior número de resultados. Na figura 1 é apresentado um diagrama de Venn que permite analisar de forma clara os resultados para cada busca, considerando todas as intersecções possíveis entre os termos. 


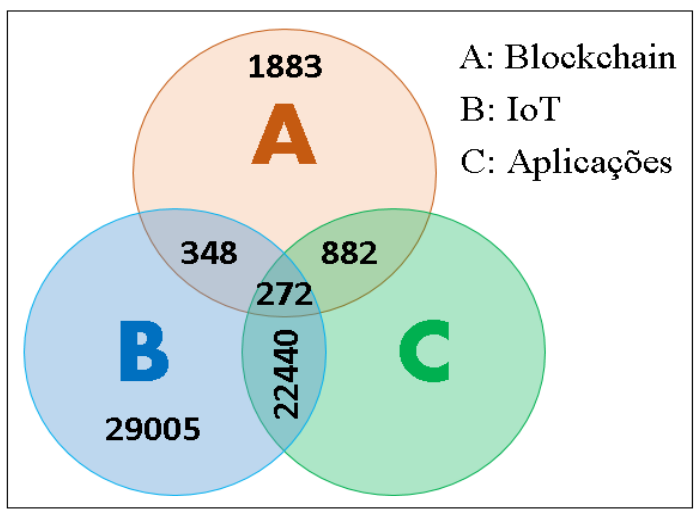

Figura 1: Diagrama de Venn com a quantidade de trabalhos encontrados na base Scopus ${ }^{\circledR}, 2019$

Fonte: Elaborado pelos autores

Mediante os resultados obtidos através de cada busca pode-se perceber que os tesauros B (IoT) e C (Aplicações) quando pesquisados são os que fornecem um maior número de resultados. A intersecção entre os tesauros A (Blockchain), B (IoT) e C (Aplicações) geram poucos resultados, pois se trata de uma busca bem mais refinada.

\subsection{ANÁLISE DA SÉRIE TEMPORAL}

É possível analisar as tendências e perspectivas de crescimento dos estudos em relação ao tema. Por isso, foi realizado um levantamento do número de trabalhos encontrados para cada ano, de acordo com cada uma das buscas. Após isso foram elaborados gráficos com base nesses resultados, objetivando promover uma melhor visualização e comparação dos dados.

$\mathrm{Na}$ figura 2, é mostrado o gráfico de evolução da quantidade de publicações de acordo com o tempo, considerando os resultados por ano encontrados para as buscas relacionadas aos Tesauros A (blockchain), B (IoT) e BC (aplicações de IoT). Essas buscas foram agrupadas no mesmo gráfico devido a seu número de resultados estarem em ordens de grandezas próximas.

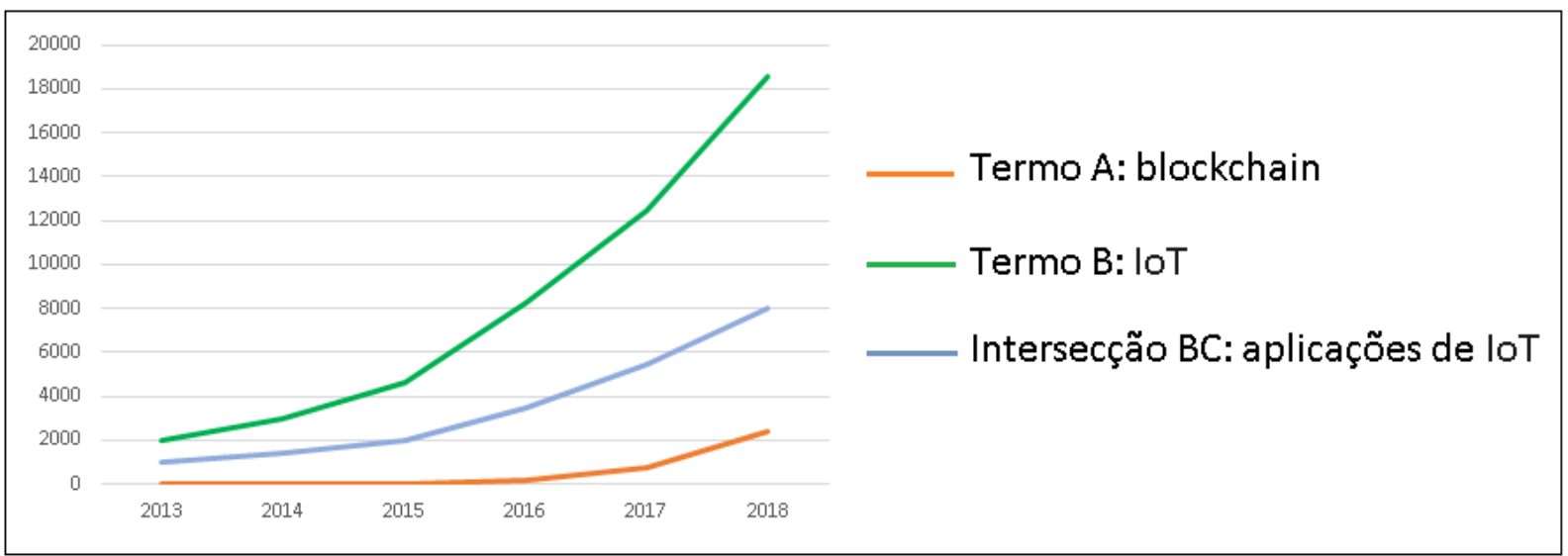

Figura 2: Evolução do número de resultados encontrados para os termos $\mathrm{A}, \mathrm{B}$ e intersecção BC

Fonte: Elaborado pelos autores

A primeira publicação de trabalhos relacionados ao Blockchain ocorreu em 2003, tendo sido publicado 3.385 trabalhos relacionados ao assunto até o ano de 2018. Com relação às pesquisas relacionadas à Internet das Coisas, a primeira publicação encontrada 
data do ano de 1934. O total de resultados encontrados foi de 52.065, sendo bem mais alto que o total encontrado para a Blockchain.

Com relação à busca realizada utilizando a intersecção $\mathrm{BC}$, foram encontrados 22.712 trabalhos, um número bem maior do que para o próprio Blockchain. O trabalho mais antigo de aplicações de IoT data do ano de 1970. Pode-se verificar portanto que a IoT é um assunto bem mais antigo e já bem mais trabalhado do que a Blockchain, que desponta como um assunto bastante contemporâneo.

$\mathrm{Na}$ figura 3 é mostrado o gráfico relacionado aos resultados encontrados para os últimos anos utilizando as intersecções AB (blockchain e IoT), AC (aplicações de blockchain) e ABC (aplicações de IoT em blockchain).

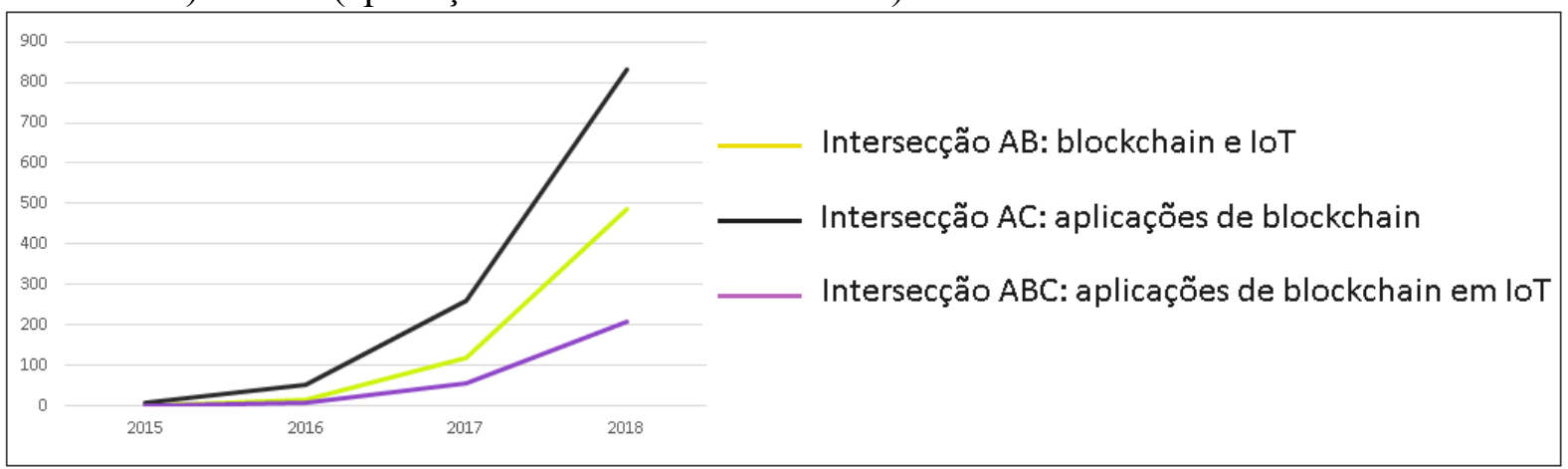

Figura 3: Evolução dos número de resultados encontrados para as intersecções $A B, A C$ e $A B C$

Fonte: Elaborado pelos autores

Como pode-se perceber, o número de resultados encontrados por essas buscas são bem menores que as anteriores, devido ao fato de se tratar de pesquisas mais direcionadas. A intersecção $\mathrm{AB}$ por exemplo, busca encontrar trabalhos que discorram concomitantemente sobre Blockchain e IoT e através dos resultados, pode-se constatar que foi encontrado um total de 620, tendo sido a maior parte desses trabalhos publicados no ano de 2018. Com relação às aplicações de Blockchain (intersecção $\mathrm{AC}$ ) o número total de trabalhos publicados é maior: 1.154. Pode-se verificar que os primeiros trabalhos sobre aplicações de Blockchain começaram a surgir em 2015, doze anos após aparecerem as primeiras publicações sobre Blockchain. A partir daí, a quantidade de trabalhos foi aumentando, o que confirma os estudos de Mirshra et al. (2016) que afirma que a trajetória de pesquisas a respeito do assunto crescerá exponencialmente ao longo de seus primeiros anos

Por fim, a busca considerando-se a intersecção entre as três palavras-chave principais do tema, resulta em uma pesquisa ainda mais específica e direcionada do que todas as anteriores. O resultado é bem menor, pois são contabilizados somente os trabalhos que discorrem especificamente sobre o tema aplicações de Blockchain na IoT. O total encontrado foi de 272 trabalhos, sendo o primeiro publicado no ano de 2016, o que leva a concluir que as aplicações de Blockchain na internet das coisas é de fato um tema muito recente.

\subsection{PRINCIPAIS AUTORES E TRABALHOS MAIS CITADOS}

Os seis principais autores encontrados em cada pesquisa, juntamente com o número de trabalhos publicados estão indicados por ordem de importância, na tabela 2. Para tal, foram considerados como mais importantes aqueles que detém maior números de trabalhos publicados para cada intersecção. 
Tabela 2: Principais autores encontrados de acordo com cada intersecção

\begin{tabular}{|c|c|c|c|c|c|c|c|}
\hline $\begin{array}{c}\text { Intersecção } \\
\mathrm{AB}\end{array}$ & $\begin{array}{c}\mathrm{N}^{\circ} \text { de } \\
\text { trabalhos }\end{array}$ & $\begin{array}{c}\text { Intersecção } \\
\mathrm{AC}\end{array}$ & $\begin{array}{c}\mathrm{N}^{\circ} \text { de } \\
\text { trabalhos }\end{array}$ & $\begin{array}{c}\text { Intersecção } \\
\mathrm{BC}\end{array}$ & $\begin{array}{c}\mathrm{N}^{\circ} \text { de } \\
\text { trabalhos }\end{array}$ & $\begin{array}{c}\text { Intersecção } \\
\mathrm{ABC}\end{array}$ & $\begin{array}{c}\mathrm{N}^{\circ} \text { de } \\
\text { trabalhos }\end{array}$ \\
\hline Deters, R. & 8 & Wang, F. Y. & 10 & Benini, L. & 43 & Fabiano, N. & 5 \\
\hline Samaniego, M. & 6 & Weber, I. & 8 & Dustdar, S. & 42 & Niyato, D. & 4 \\
\hline Fabiano, N. & 5 & Xu, X. & 8 & $\begin{array}{c}\text { Rodrigues, J. } \\
\text { J. P. C. }\end{array}$ & 37 & Wang, P. & 4 \\
\hline Jang, J. W. & 5 & Chen, L. & 6 & Moerman, I. & 35 & Cha, S. C. & 3 \\
\hline Sharma, P. K. & 5 & Gao, Z. & 6 & Fortino, G. & 33 & Guizani, M. & 3 \\
\hline Choo, K. K. R. & 4 & Shi, W. & 6 & Atzori, L. & 32 & Putisek, M. & 3 \\
\hline
\end{tabular}

Fonte: Elaborado pelos autores

Analisando-os é possível perceber que são dispersos entre si, ou seja, os principais autores encontrados para a intersecção $\mathrm{AB}$ por exemplo, não são os mesmos para a intersecção $\mathrm{ABC}$, ocorrendo muito poucas coincidências entre cada intersecção. Isso ocorre provavelmente pelo fato de ser um tema novo, por isso ainda há poucos pesquisadores na área e não houve tempo suficiente para a publicação de grandes quantidades de trabalho, o que contribui para essa dispersão.

Além dos autores responsáveis por maior número de publicações, é importante analisar também quais os trabalhos mais citados, que podem servir de apoio para sustentação de novos trabalhos quanto à revisão de literatura. Na tabela 3, seguem indicados os títulos dos trabalhos mais citados considerando a pesquisa pela intersecção $\mathrm{ABC}$, juntamente com os autores, o ano de publicação e o número de citações.

Tabela 3: Trabalhos com maior número de citações

\begin{tabular}{|c|c|c|c|}
\hline Título do Trabalho & Autor (es) & Ano & $N^{\circ}$ de citações \\
\hline $\begin{array}{c}\text { Blockchains and Smart Contracts } \\
\text { for the Internet of Things }\end{array}$ & $\begin{array}{l}\text { Christidis, K., } \\
\text { Devetsikiotis, M. }\end{array}$ & 2016 & 415 \\
\hline$\frac{\text { An Overview of Blockchain Technology: }}{\frac{\text { Architecture, Consensus, and Future }}{\underline{\text { Trends }}}}$ & $\begin{array}{c}\text { Zheng, Z., } \\
\text { Xie, S., } \\
\text { Dai, H., } \\
\text { Chen, X., } \\
\text { Wang, H. }\end{array}$ & 2017 & 133 \\
\hline Can Blockchain Strengthen Internet & Kshetri, N. & 2017 & 75 \\
\hline
\end{tabular}




\begin{tabular}{|c|c|c|c|}
\hline of Things? & & \\
\hline $\begin{array}{c}\text { Internet of Things, Blockchain and Shared } \\
\text { Economy Applications }\end{array}$ & $\begin{array}{c}\text { Huckle, S. } \\
\text { Bhattacharya, R. } \\
\text { White, M. } \\
\text { Beloff, N. }\end{array}$ & 2016 & 71 \\
\hline$\underline{\text { Towards an optimized blockchain }}$ for IoT & $\begin{array}{c}\text { Dorry, A. } \\
\text { Kanhere, S. S. } \\
\text { Jurdak, R. }\end{array}$ & 2017 & 66 \\
\hline $\begin{array}{c}\text { Industry 4.0: State of the art and future } \\
\text { trends }\end{array}$ & $\begin{array}{c}\text { Xu, L. D. } \\
\text { Xu, E. L. } \\
\text { Li, L. }\end{array}$ & 2018 & 62 \\
\hline
\end{tabular}

Fonte: Elaborado pelos autores

Analisando-se a tabela, pode-se perceber que curiosamente os autores do trabalho mais citado não figuram entre aqueles que detém maior quantidade de publicações relacionadas ao tema. Isso deve ter acontecido pelo fato de esses autores terem publicado uma quantidade menor de trabalhos relacionados ao tema, enquanto outros autores publicaram maior quantidade de trabalhos relacionados a essa temática, no entanto não obtiveram muitas citações. É provável que isso aconteça devido ao baixo número de publicações a respeito do assunto. Segundo Ferreira et al. (2017), a tecnologia e as pesquisas a respeito ainda estão em desenvolvimento, o que pode fazer com que autores mais citados não sejam necessariamente os que possuem maior quantidade de trabalhos relacionados ao assunto.

\subsection{DIVISÕES POR ÁREAS TEMÁTICAS}

As áreas ou setores de pesquisa que tiveram maior número de publicações de trabalhos envolvendo a Blockchain e a IoT encontram-se em ordem decrescente de publicações na tabela 4.

Tabela 4: Áreas de pesquisa responsáveis por maior número de publicações sobre IOTe Blockchain

\begin{tabular}{|c|c|}
\hline Blockchain & IOT \\
\hline Ciência da Computação & Ciência da Computação \\
\hline Engenharia & Engenharia \\
\hline Matemática & Matemática \\
\hline Ciência das Decisões & Física e Astronomia \\
\hline
\end{tabular}




\begin{tabular}{|c|c|}
\hline $\begin{array}{c}\text { Negócios, Gestão e } \\
\text { Contabilidade }\end{array}$ & Ciência das Decisões \\
\hline Ciências Sociais & Ciências Sociais \\
\hline
\end{tabular}

Fonte: Elaborado pelos autores

Com relação às pesquisas para cada intersecção, na tabela 5 encontram-se indicadas as áreas temáticas em ordem decrescente do número de publicações.

Tabela 5: Áreas de pesquisas responsáveis por maior número de publicações, para cada intersecção

\begin{tabular}{|c|c|c|c|}
\hline \multirow{2}{*}{ Intersecção } & A, B & A, C & B, C \\
\hline Principais & $\begin{array}{c}\text { Ciência da } \\
\text { Computação } \\
\text { Aloçockes de }\end{array}$ & $\begin{array}{c}\text { Ciência da } \\
\text { Computação }\end{array}$ & $\begin{array}{c}\text { Ciência da } \\
\text { Computação }\end{array}$ \\
\hline Áreas: & Engenharia & Engenharia & Engenharia \\
\hline & Ciência das Decisões & Matemática & Matemática \\
\hline & Matemática & Ciência das Decisões & Física e Astronomia \\
\hline & $\begin{array}{c}\text { Negócios, Gestão e } \\
\text { Contabilidade }\end{array}$ & $\begin{array}{c}\text { Negócios, Gestão e } \\
\text { Contabilidade }\end{array}$ & Ciências Sociais \\
\hline & Ciências Sociais & Ciências Sociais & $\begin{array}{c}\text { Ciências dos } \\
\text { Materiais }\end{array}$ \\
\hline
\end{tabular}

Fonte: Elaborado pelos autores

Para o caso da intersecção ABC foi realizada uma análise mais apurada das áreas temáticas, pelo fato de se tratar da pesquisa principal.

$\mathrm{Na}$ figura 4, é mostrado um gráfico que retrata a proporção das áreas temáticas responsáveis por maior número de publicações de trabalhos relacionados ao tema. 


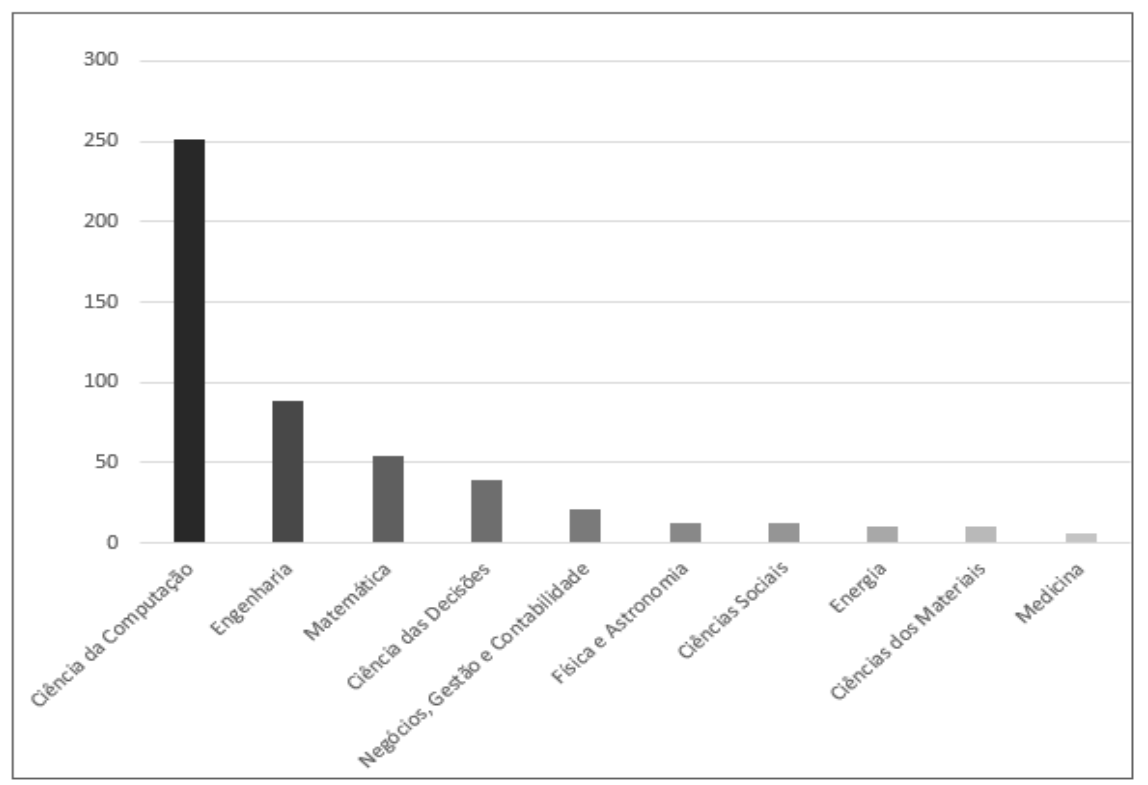

Figura 4: Principais áreas temáticas relacionadas ao tema

Fonte: Elaborado pelos autores

De acordo com o gráfico pode-se perceber um grande predomínio de publicações a respeito do tema na área de ciência da computação. Dos 272 trabalhos encontrados, um total de 251 está relacionado a essa área, sendo importante destacar o fato de que um mesmo trabalho pode pertencer a mais de uma área temática. A área de Engenharia apresentou 89 trabalhos, seguida pelas áreas de Matemática e Ciências das Decisões, com respectivamente 89 e 54 trabalhos.

\subsection{PRINCIPAIS PAÍSES, AFILIAÇÕES E TIPOS DE DOCUMENTOS RELACIONADOS}

Tendo como base a pesquisa realizada utilizando a intersecção $\mathrm{ABC}$, pode-se determinar quais são os principais países, afiliações e tipos de documento que mais se relacionam ao tema aplicações de Blockchain em IoT.

Na figura 5 é mostrado um gráfico comparativo de publicações por países/territórios. 


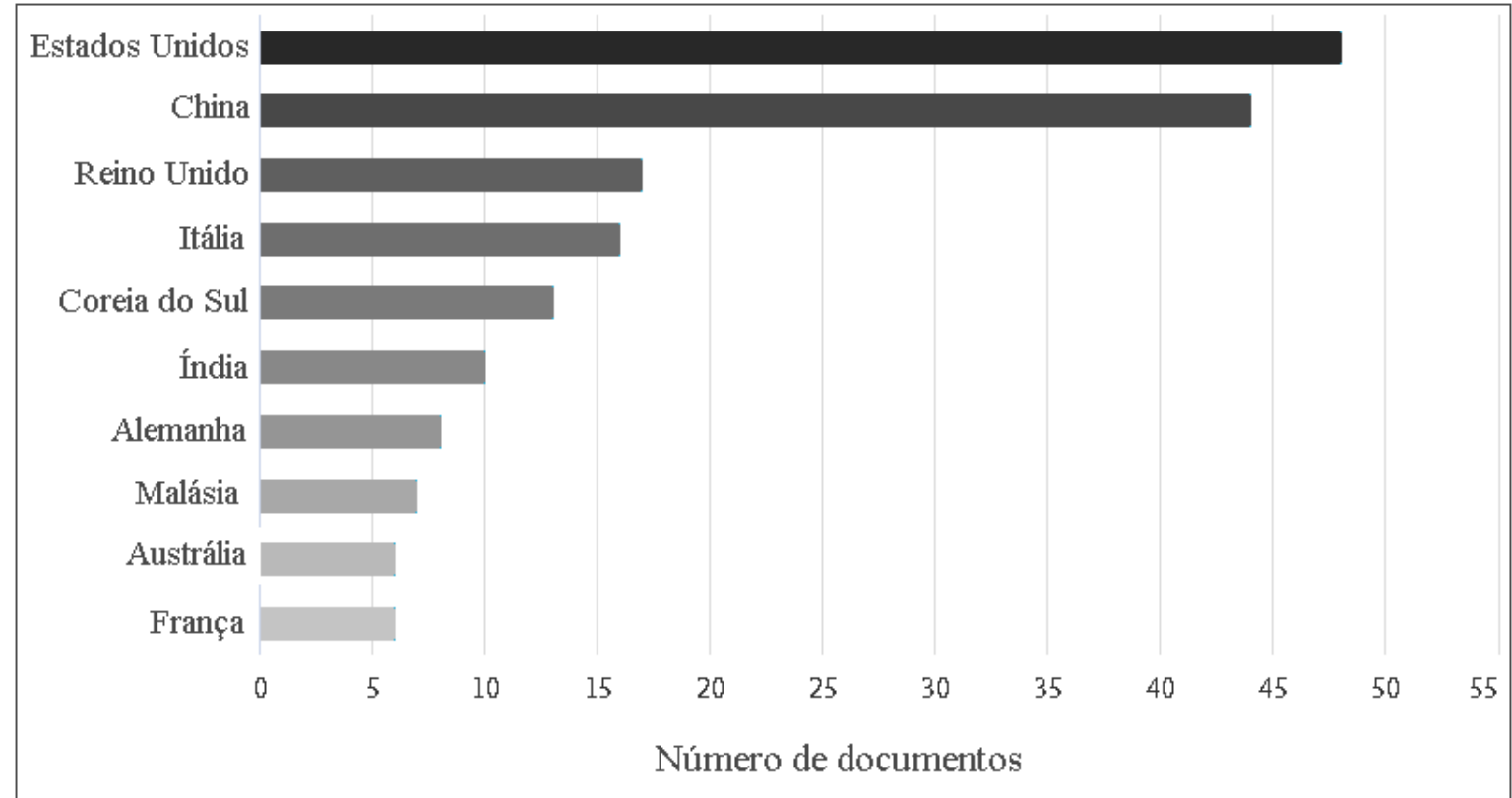

Figura 5: Número de publicações por países

Fonte: Elaborado pelos autores

De acordo com o gráfico pode-se constatar que os Estados Unidos são o país responsável por desenvolver maior número de trabalhos relacionados ao tema, seguido bem de perto pela China e depois pelo Reino Unido.

Além de identificar os países líderes com relação às publicações na área, é importante também verificar quais são as afiliações mais influentes. Na figura 6, destacam-se as principais afiliações ou fontes de pesquisa.

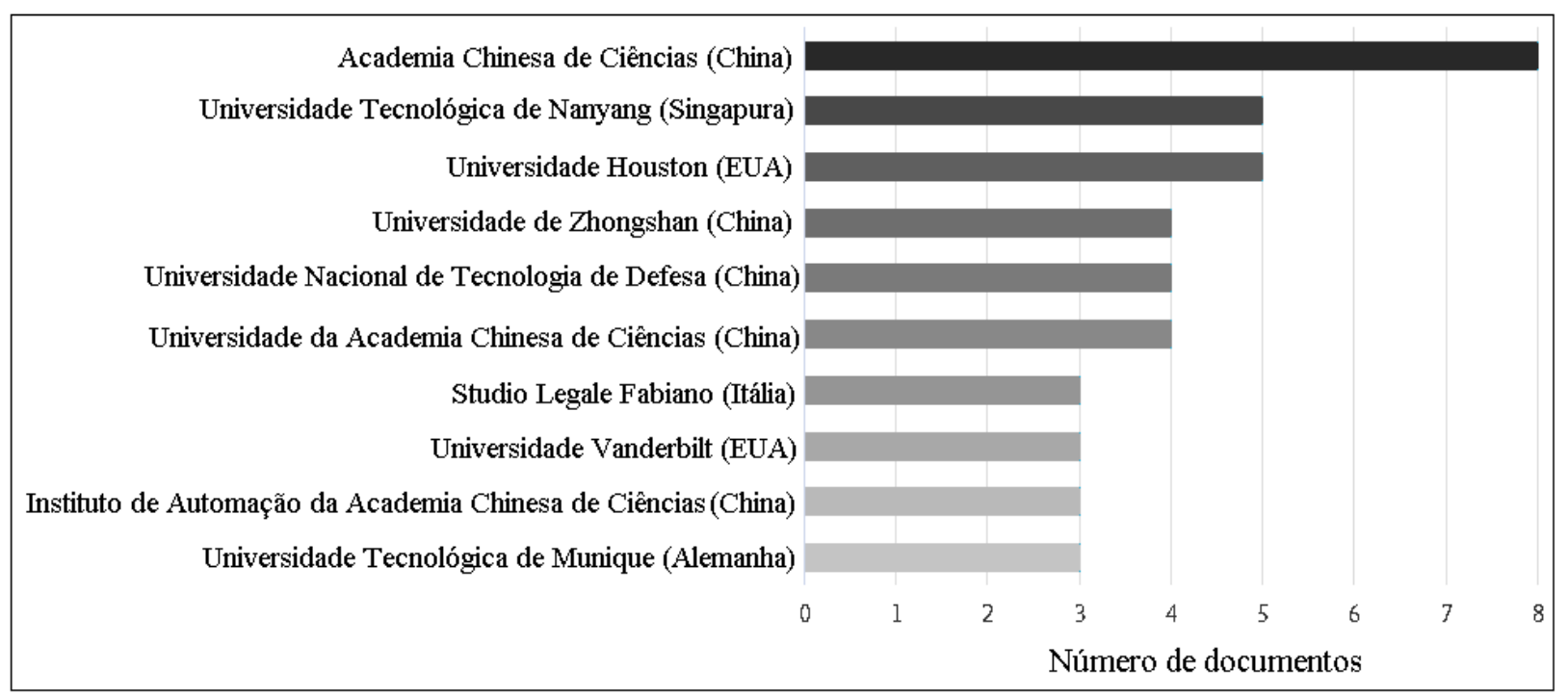

Figura 6: Principais afiliações

Fonte: Elaborado pelos autores 
Apesar de os Estados Unidos serem o país que detém maior influência com relação às pesquisas dessa área, pode-se destacar também uma grande influência da China, uma vez que cinco das dez principais afiliações são chinesas, enquanto apenas duas são americanas. Isso provavelmente ocorreu porque algumas afiliações chinesas centralizaram as publicações relacionadas ao tema, enquanto que nos Estados Unidos, apesar de possuir uma quantidade maior de publicações, elas encontram-se dispersas entre suas afiliações.

Quanto aos tipos de documentos, é possível verificar os mais relacionados ao assunto através da figura 7.

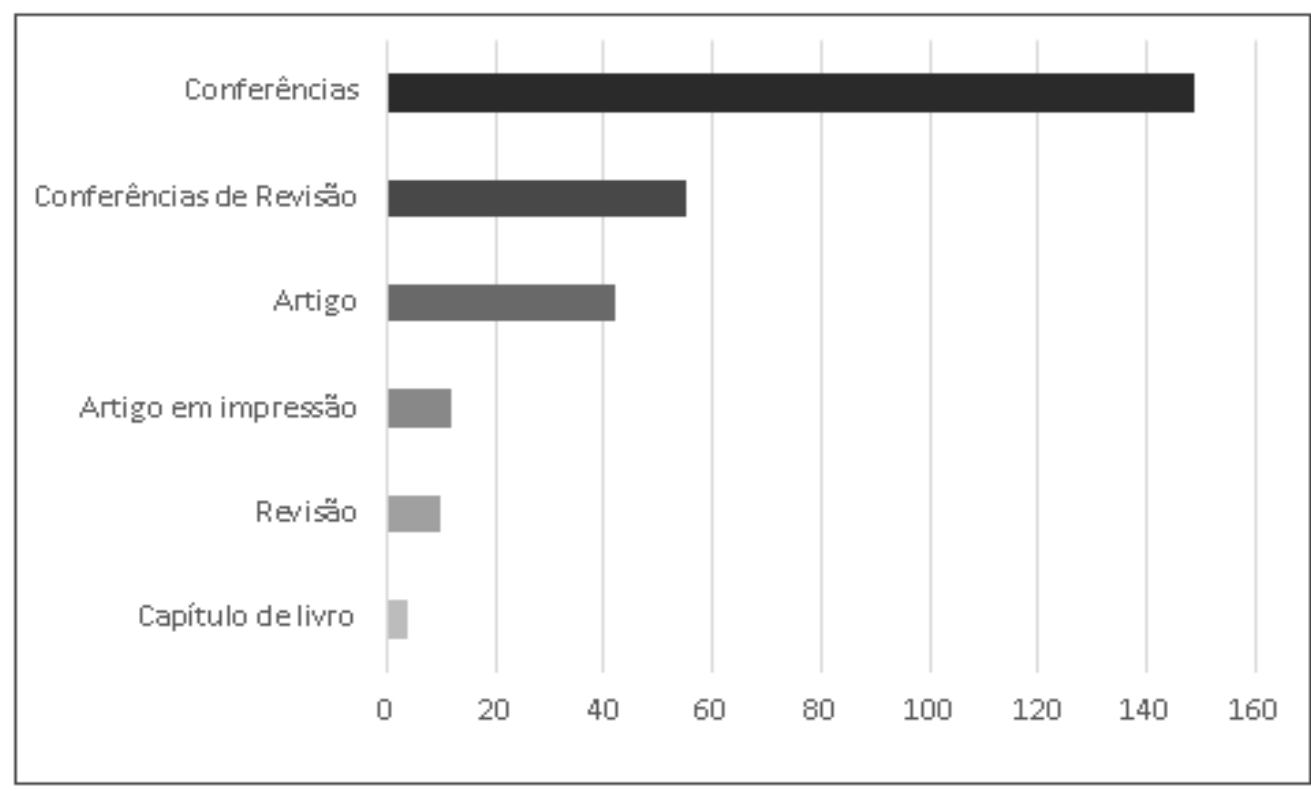

Figura 7: Principais documentos relacionados ao tema

Fonte: Elaborado pelos autores

Pode-se observar que a maioria das publicações relacionadas ao tema advêm de conferências que detém $54,8 \%$. Um pouco atrás aparecem as conferências de revisão $(20,2 \%$ do total) e os artigos com 15,4\%.

\section{CONSIDERAÇÕES FINAIS}

Com base em tudo o que foi apresentado e discutido neste presente trabalho, pode-se concluir que as aplicações de Blockchain em IoT são um tema recente, e apresentam grande potencial para trazer benefícios com relação ao aumento da segurança da informação. As publicações se iniciaram em 2016, treze anos após ter surgido a primeira pesquisa sobre Blockchain, que data do ano de 2003. Apresenta tendências de crescimento ao longo dos anos, o que indica se tratar de um tema promissor. A maior parte dos trabalhos encontrados sobre Blockchain, IoT e suas aplicações foram publicados nos últimos anos (2017 e 2018). Entre todas as buscas realizadas nessa pesquisa, o trabalho mais antigo identificado é do ano de 1934, correspondente aos trabalhos encontrados sobre IoT. Pode-se perceber que, apesar do fato de a maior parte de seus estudos se concentrar nos últimos anos, o conceito de IoT não é tão recente, visto a data da primeira publicação encontrada, ao contrário da Blockchain, que por sua vez é um conceito bem mais novo, tendo ocorrido em 2003 a primeira publicação de um trabalho relacionado ao assunto. Pode-se concluir que o tema é bastante multidisciplinar, podendo ser empregado em diversos campos. Apresenta maior 
aplicabilidade com relação à área da ciência da computação, seguido pela engenharia e pela matemática, porém também pode ser utilizado em outras diversas áreas, tais como, ciência das decisões, ciências sociais, contabilidade, ciências dos materiais, etc.

Tendo em vista a importância dessa temática, o trabalho realizado pode servir como base para orientar pesquisadores e estudiosos que pretendam explorá-la, oferecendo uma boa compreensão das tendências de evolução, principais autores, áreas de pesquisa, trabalhos mais citados, filiações mais importantes, entre outras informações que abarcam o tema. Pode-se citar como sugestão para trabalhos futuros a realização deste estudo utilizando outras bases de dados, para que possam ser comparados os resultados, de forma a oferecer uma percepção ainda melhor e mais precisa a respeito das tendências e do estado em que se encontram os estudos acerca do assunto escolhido para análise.

\section{REFERÊNCIAS}

CHICARINO, V. R.; JESUS, E. F.; ALBUQUERQUE, C. V. N.; ROCHA, A. A. Uso de blockchain para privacidade e segurança em internet das coisas. Livro de Minicursos do VII Simpósio Brasileiro de Segurança da Informação e de Sistemas Computacionais. Brasília: SBC, 2017.

COSTA, H. G. Modelo para webibliomining: proposta e caso de aplicação. Revista da FAE, v. 13, n. 1, p. 115-126, 2010.

EVANS, D. The Internet of Things: How the Next Evolution of the Internet Is Changing Everything. CISCO white paper, v. 1, p. 1-11, 2011. Disponível em:

$<$ https://www.cisco.com/c/dam/en_us/about/ac79/docs/innov/IoT_IBSG_0411FINAL.pdf.> Acesso em: maio 2018 .

FERNÁNDEZ-CARAMÉS, T. M.; FRAGA-LAMAS, P. A Review on the Use of Blockchain for the Internet of Things. IEEE Access, p. 1-1, 2018.

FERREIRA, J. E., PINTO, F. G. C., SANTOS, S. C. Estudo de mapeamento sistemático sobre as tendências e desafios do Blockchain. Revista Gestão.Org, v. 15, Edição Especial, p. 108-117 ISSN 1679-1827, 2017.

HÖLLER, J. (ED.). From machine-to-machine to the Internet of things: introduction to a new age of intelligence. Amsterdam: Elsevier Academic Press, 2014.

JEON, J. H.; KIM, K. H.; KIM, J. H. Block chain based data security enhanced IoT server platform. In: 2018 INTERNATIONAL CONFERENCE ON INFORMATION NETWORKING (ICOIN), 2018

JESUS, E. F.; CHICARINO, V. R. L.; AlbUQUERQUE, C. V. N.; ROCHA, A. A. de A. A Survey of How to Use Blockchain to Secure Internet of Things and the Stalker Attack. Security and Communication Networks, v. 2018, p. 1-27, 2018.

KYPRIOTAKI, K.; ZAMANI, E.; GIAGLIS, G. From Bitcoin to Decentralized Autonomous Corporations Extending the Application Scope of Decentralized Peer-to-Peer Networks and Blockchains: SCITEPRESS Science and and Technology Publications, 2015Disponível em:

$<$ http://www.scitepress.org/DigitalLibrary/Link.aspx?doi=10.5220/0005378402840290>. Acesso em: 24 maio. 2018

LEAL, R. L. V. Blockchain e Internet das coisas: Aplicações e Iniciativas. p. 33, 2017. 
LEE, C. H.; KIM, K. H. Implementation of IoT system using block chain with authentication and data protection. 2018 International Conference on Information Networking (ICOIN). Anais... In: 2018 INTERNATIONAL CONFERENCE ON INFORMATION NETWORKING (ICOIN). jan. 2018

LOPES, S.; COSTA, M. T.; LLIMÓS, F. F.; AMANTE, M. J.; LOPES, P. F. A Bibliometria e a Avaliação da Produção Científica: indicadores e ferramentas. Actas do Congresso Nacional de Bibliotecários, Arquivistas e Documentalistas, v. 0, n. 11, 18 out. 2012.

LUCENA, A. U. DE; HENRIQUES, M. A. A. Estudo de arquiteturas dos blockchains de Bitcoin e Ethereum. 2016.

MiShra, D., GUNASEKARAN, A., CHILde, S. J., PAPADOPOUlos, T., DUBEY, R., WAMBA, S. Vision, applications and future challenge of Internet of Things: A bibliometric study of the recente literature. Emerald Group Publishing Limited, Volume 116, ages 1331-1355, 2016.

NOVO, O. Blockchain Meets IoT: An Architecture for Scalable Access Management in IoT. IEEE Internet of Things Journal, v. 5, n. 2, p. 1184-1195, abr. 2018.

OLIVEIRA, E. F. T. DE; GRÁCIO, M. C. C. Visibilidade dos pesquisadores no periódico Scientometrics a partir da perspectiva brasileira: um estudo de cocitação. Em Questão, v. 18, n. 3, p. 99-113, 10 jan. 2013.

RAVELLI, A. P. X., FERNANDES, G. C. M., BARBOSA, S. F. F., SiMÃO, E. SANTOS, S. M. A., MEIRELLES, B. H. S. A produção do conhecimento em enfermagem e envelhecimento: um estudo bibliométrico. Revista Texto e Contexto Enfermagem, Florianópolis, 2009.

REDONDO, M.; LEON, L.; POVEDANO, F. J.; ABASOLO, L.; PEREZ-NIETO, M.A.; LOPEZMUÑOZ, F. A bibliometric study of the scientific publications on patient-reported outcomes in rheumatology. Seminars in Arthritis and Rheumatism, v. 46, n. 6, p. 828-833, 1 jun. 2017.

XU, Q.; AUNG, K. M. M.; ZHU, Y.; YONG, K. L. A Blockchain-Based Storage System for Data Analytics in the Internet of Things. In: YAGER, R. R.; PASCUAL ESPADA, J. (Eds.). . New Advances in the Internet of Things. Cham: Springer International Publishing, 2018. v. 715p. 119-138. 